\title{
Unsatisfied relatedness, not competence or autonomy, increases trait anger through the right amygdala
}

\author{
Yinan Wang ${ }^{1}$ • Feng Kong ${ }^{2}$ - Xiangzhen Kong ${ }^{2}$ - Yuanfang Zhao ${ }^{2}$ - Danhua Lin ${ }^{1}$. \\ Jia Liu ${ }^{1,2}$
}

Published online: 12 June 2017

(C) Psychonomic Society, Inc. 2017

\begin{abstract}
Anger is a common negative emotion in social life. Behavioral research suggests that unsatisfied relatedness, autonomy, and competence are related to anger. However, it remains unclear whether these unsatisfied needs all contribute to anger or just a particular unsatisfied need is the main source of anger. In addition, little is known about the neural substrate between unsatisfied needs and anger. To address these two questions, we used voxel-based morphometry (VBM) to explore the neural substrate underlying the relation between unsatisfied needs and trait anger. Behaviorally, we found that although all three unsatisfied needs were correlated with trait anger, unsatisfied relatedness was the only factor that was uniquely related to trait anger. Neurally, the gray matter volume of the right amygdala was correlated with trait anger, which fits nicely with the role of the amygdala as a core region for processing anger. Importantly, the right amygdala mediated the total effect of unsatisfied relatedness on trait anger, even after controlling for general personality dispositions. Our results contribute to the theoretical conceptualization of anger by elucidating the unique role of unsatisfied relatedness in anger and the neural substrate underlying such relation.
\end{abstract}

Electronic supplementary material The online version of this article (doi:10.3758/s13415-017-0523-y) contains supplementary material, which is available to authorized users.

Jia Liu

liujia@bnu.edu.cn

1 School of Psychology, Beijing Key Laboratory of Applied Experimental Psychology, Jia Liu, Room 405, Yingdong Building, 19 Xinjiekouwai St, Haidian District, Beijing 100875, China

2 State Key Laboratory of Cognitive Neuroscience and Learning \& IDG/McGovern Institute for Brain Research, Beijing Normal University, Beijing, China
Keywords Need satisfaction · Anger · Amygdala Voxel-based morphometry (VBM)

Anger is a universal emotion, which is generally regarded as a negative emotion (Harmon-Jones, 2004) because it is elicited by unpleasant or undesired events. Since these events may occur for a variety of reasons (Carver \& Harmon-Jones, 2009), there are inconsistent views regarding the causes of anger. It is often assumed that people tend to become angry when they possess low competence, for instance, which keeps them from reaching their goals (Roseman \& Smith, 2001; Smith, Haynes, Lazarus, \& Pope, 1993). In addition, some emotion theorists propose that anger is a reaction to the violation of autonomy (individual freedom or rights; Fischer \& Roseman, 2007), because anger is associated with behaviors aiming at removing an obstacle and asserting control (Frijda, Kuipers, \& Ter Schure, 1989). A third possible cause is that anger is elicited by interpersonal rejection because people often become angry when they feel devalued, unincluded, or outright rejected (Leary, Twenge, \& Quinlivan, 2006).

In nature, the three plausible causes of anger reflect the human beings' three basic psychological needs: competence, autonomy, and relatedness (Deci \& Ryan, 2000), which can result in negative affective consequences when they are not satisfied (Sheldon \& Gunz, 2009). The frustration-aggression hypothesis proposes that all aggression or anger could be traced to one or more prior frustrations, and the strength of the resulting anger would be in direct proportion to the strength of the nonfulfilled desires (Dollard, Miller, Doob, Mowrer, \& Sears, 1939). Conversely, the inability to achieve the desired and expected goal would intensify individuals' aggressive tendencies (Berkowitz, 1989). Hence, the nonfulfilled desires or unsatisfied needs might be more pivotal in provoking 
anger. However, it remains unclear whether the three needs have a unique contribution to anger when unsatisfied or if a specific unsatisfied need is the major source of anger. Furthermore, little is known about how unsatisfied needs exert an effect on anger.

The neural substrate underlying the relation need satisfaction and anger identified in this study may help clarifying how need satisfaction influences anger. Previous neuroimaging studies have suggested that the amygdala, a limbic structure involved in processing emotion and potentially threatening stimuli, is a core region for the processing of anger (Reuter, Weber, Fiebach, Elger, \& Montag, 2009; Rüsch et al., 2003). For example, there is a negative correlation between trait anger score and the volume of the left amygdala (Reuter et al., 2009), and trait anger is found to be positively correlated with the neural activity of the amygdala when viewing angry facial expressions (Beaver, Lawrence, Passamonti, \& Calder, 2008; Coccaro, McCloskey, Fitzgerald, \& Phan, 2007). These findings suggest an intrinsic link between the amygdala and anger.

Meanwhile, a recent study suggests the right and left amygdala are involved in processing different types of fearful faces (Carlson, Greenberg, \& Mujica-Parodi, 2010). Specifically, individuals who score higher in anger expression have increased neural activity in the left amygdala to crude representations of fearful faces, which may reflect a mechanism triggering aggressive behaviors. In contrast, trait anger negatively covaried with the right amygdala response to detailed fear expressions. In other words, the left amygdala is more related to antisocial or aggressive behaviors (Blair, 2003), while the right amygdala is more related to trait anger and fear processing (Carlson et al., 2010). Because of its functional dissociation between the left and right amygdala, the hemispheric asymmetry of the amygdala in the relation between unsatisfied needs and trait anger, if any, may help elucidate its role in aggression (i.e., approach-related behaviors) or fear (i.e., withdrawal behaviors).

To explore the neural substrate between unsatisfied needs and trait anger, the current study used voxel-based morphometry (VBM) to examine whether and how unsatisfied needs were correlated with trait anger through the right, left, or both amygdala. Trait anger was measured using the Novaco Anger Scale, Part B (Novaco, 1994), which measures anger intensity across a range of potentially provoking situations. Need satisfaction was assessed using the Balanced Measure of Psychological Needs (Sheldon \& Gunz, 2009; Sheldon \& Hilpert, 2012), which measures the degree of satisfaction for the three basic psychological needs: autonomy, competence, and relatedness. We aimed to discover (1) whether unsatisfied needs of autonomy, competence, or relatedness had a unique contribution to trait anger and (2) whether unsatisfied needs exerted an effect on trait anger through the amygdala.

\section{Materials and Method}

\section{Subjects}

Fifty-seven college students (20 males; mean age \pm standard deviation $=22.72 \pm 3.51$ years) participated in this study. All participants reported no past or current psychiatric illness or history of neurological disorders (e.g. epilepsy, traumatic brain injury, neurodegenerative disorders, or cerebrovascular diseases), no intellectual disability, and no significant systemic medical illness. The Institutional Review Board of Beijing Normal University approved both the behavioral and MRI protocols. Written informed consent was obtained from all participants prior to the experiment.

\section{Anger measure}

The 25-item Novaco Anger Scale, Part B (Novaco, 1994) is a self-reported scale indexing the frequency with which individuals generally perceive encountered situations to arouse their anger (e.g., "You unpack an appliance that you just bought, plug it in, and discover that it doesn't work"). It has five subscales (Disrespectful Treatment, Unfairness/Injustice, Frustration/Interruption, Annoying Traits, and Irritations), and the responses are made on a 5-point scale measuring intensity, ranging from 1 (feel little or no annoyance) to 5 (feel very angry). Because individuals' general anger intensity across a range of potentially provoking situations reflects their level of trait anger (Mills, Kroner, \& Forth, 1998), the ratings of the subscales were combined (Cronbach's $\alpha=.94$ ).

\section{Unsatisfied need measure}

The 18-item Balanced Measure of Psychological Needs (Sheldon \& Gunz, 2009; Sheldon \& Hilpert, 2012) containing six items each for autonomy, competence, and relatedness was used to assess the satisfaction of participants' needs. According to the self-determination theory (Deci \& Ryan, 2000; Sheldon \& Hilpert, 2012), autonomy is defined as the experience of volition, choice, and self-regulation, with sample items including "I was free to do things my own way" (positive) or "I was lonely" (negative). Competence is defined as the experience of effectance, mastery, and ability, with sample items including "I was successfully completing difficult tasks and projects" (positive) or "I experienced some kind of failure, or was unable to do well at something" (negative). Relatedness is defined as the experience of support, connection, and closeness with important others, with sample items including "I felt a sense of contact with people who care for me and whom I care for" (positive) or "There were people telling me what I had to do" (negative). The Balanced Measure of Psychological Needs was administered with a 5point scale: 1 (no agreement) to 5 (much agreement). 
Positively worded items were reversed so that higher scores reflected lower need satisfaction. Autonomy, Competence, and Relatedness subscales all showed high internal consistency (Cronbach's $\alpha=.80, .88$, and .71, respectively).

\section{Potential confounds}

We assessed several demographic, neurobiological, and psychosocial characteristics that might provide alternative explanations for any observed associations, and then modeled them as covariates. The demographic covariates included age and sex. The neurobiological covariate referred to the total gray matter volume (GMV) of the whole brain. Considering that both age and sex are found to be significant determinants of the interindividual brain morphology variability (Ge et al., 2002; Lemaitre et al., 2005; Tisserand et al., 2004), we included age and sex as well as whole-brain GMV as confounding covariates in our analysis. We also included Big-Five personality traits and positive and negative affection as psychosocial covariates. Participants' personality was assessed using the Ten Item Personality Inventory (Gosling, Rentfrow, \& Swann, 2003), which measures individuals' Big-Five personality traits. Last, positive affect (PA) and negative affect (NA) were measured using the Positive and Negative Affect Schedule (Watson, Clark, \& Tellegen, 1988). The PA scale included 10 items such as interested, and the NA scale included 10 items such as upset. Participants were instructed to estimate the extent to which they generally experienced these emotions. Cronbach's alpha for PA and NA was .93 and .89, respectively.

\section{MRI data acquisition}

Scanning was conducted on a Siemens $3 \mathrm{~T}$ scanner (MAGENTOM Trio, a Tim system) with a 12-channel phased-array head coil at BNU Imaging Center for Brain Research, Beijing, China. A 3-D structural MRI was acquired on each subject using a T1-weighted MPRAGE sequence $(\mathrm{TR} / \mathrm{TE} / \mathrm{TI}=2530 / 3.39 / 1100 \mathrm{~ms}$, flip angle $=7$ degrees, FOV $=256 \times 256 \mathrm{~mm}^{2}$ ). One hundred and twenty-eight contiguous sagittal slices were imaged with $1 \times 1 \mathrm{~mm}$ in-plane resolution and $1.33 \mathrm{~mm}$ slice thickness for whole-brain coverage.

\section{Voxel-based morphometry (VBM) analysis}

VBM analysis was employed to quantify gray matter (GM) in each voxel across the whole brain (Ashburner \& Friston, 2000). Specifically, VBM was performed using SPM8 software (http://www.fil.ion.ucl.ac.uk/spm) and the DARTEL toolbox with default settings for all parameters unless explicitly stated. First, image quality was assessed by visual examination, and no participant was excluded due to scanner artifacts or gross anatomical abnormalities. Second, the origin of the spatial coordinates was manually set to the anterior commissure for each participant. Third, images were segmented into four distinct tissue classes-GM, white matter, cerebrospinal fluid, and other tissue - using a unified segmentation approach (Ashburner \& Friston, 2005). Fourth, the segmented GM images were normalized to the GM map provided in SPM, and resampled to 2-mm isotropic voxels. Fifth, the images were nonlinearly registered with DARTEL, which involves iteratively generating a study-specific template based on the tissue maps from all participants, and then warping all participants' GM images onto the generated template to increasingly improve alignment (Ashburner, 2007). Sixth, the GM images were normalized into standard MNI space, and voxel values in an individual's GM images were modulated by multiplying the Jacobian determinants derived from registration (Good et al., 2002). Finally, the modulated GM images were then smoothed with an 8-mm full-width at half-maximum (FWHM) isotropic Gaussian kernel. The preprocessed GM images were used for further analyses.

\section{Statistical analysis}

First, we investigated whether unsatisfied relatedness, competence, and autonomy were associated with trait anger by calculating the Pearson product-moment correlation coefficient. Then, to test whether any of the three unsatisfied needs made a unique predictive contribution to trait anger or if one of them played a leading role in predicting anger, we conducted a multiple-regression analysis. The scores for relatedness, competence, and autonomy satisfaction were entered simultaneously into the regression equation to predict trait anger; meanwhile, age and sex were also included as covariates. Furthermore, to examine whether the Novaco Anger Scale was biased toward poor social relations, we computed correlations between each item of the scale and three unsatisfied needs.

Second, to examine whether trait anger was associated with individuals' amygdala GMV as seen in a previous study (Reuter et al., 2009), we conducted a region of interest (ROI) analysis. The ROIs for right and left amygdala were defined using probabilistic maps from the Harvard-Oxford Subcortical Structural Atlas, available for FMRIB Software Library (FSL), with a threshold of 50\%. That is, only voxels that showed a $50 \%$ or greater probability of being labeled as a corresponding structure were included. Partial correlation analyses were performed to determine whether the right or left amygdala GMV showed significant correlations with trait anger, when controlled for age, sex, and whole-brain GMV.

Third, after finding that trait anger was associated with an individuals' right or left amygdala GMV, we performed mediation analysis using the SPSS macro programmed by Preacher and Hayes (2008) to examine whether the GMV of 
that region mediated the relationship between the unsatisfied need and trait anger. In this analysis, a bootstrapping approach was used to test the significance of the indirect effect of the independent variable (IV, i.e., need satisfaction) on the dependent variable (DV, i.e., trait anger) through the mediator (M, i.e., amygdala GMV). In mediation analysis, the total effect (Path c) refers to the relationship between the IV and DV without controlling for the $\mathrm{M}$. The direct effect (Path $\mathrm{c}^{\prime}$ ) refers to the relationship between the IV and the DV after controlling for the M. An estimate of the indirect effect was derived from the mean of 5,000 bootstrap samples, bias was corrected, and an accelerated $95 \%$ confidence interval was calculated (Preacher \& Hayes, 2008). If the empirical 95\% confidence interval does not include zero, the indirect effect is statistically significant (i.e., $p<.05$ ). To test whether these results were specific and reliable, we also examined the mediating effect of amygdala GMV between need satisfaction and trait anger after controlling for several other psychological measures, including Big-Five personality traits and positive and negative affect.

Finally, we conducted a whole-brain voxel-wise analysis using a general linear model to explore other regions outside of the amygdala potentially showing an association with trait anger. To remove voxels of noninterest, a threshold value of 0.2 for all GM images was used to generate a GM mask across the whole brain. Meanwhile, to identify regions to compare mediation models with the amygdala mediation models, we used a liberal threshold $(p<.05$, uncorrected).

\section{Results}

Means, standard deviations, scoring range, skewness, and kurtosis for all psychological measures are presented in Table 1. Kurtosis and skewness of all measures ranged from -1 and +1 , indicating the normality of the data (Marcoulides \& Hershberger, 1997). Furthermore, correlation analyses showed that trait anger was correlated with low relatedness satisfaction $(r=.53, p<.001)$, autonomy satisfaction $(r=.42$, $p<.01)$, and competence satisfaction $(r=.35, p<.01$; see Table 2), indicating that all three unsatisfied needs were positively associated with trait anger. To further assess whether the three unsatisfied needs were uniquely correlated with trait anger, we employed simultaneous multiple regression analyses. We found that only unsatisfied relatedness was significantly correlated with trait anger, which explained approximately $35 \%$ of the variance in trait anger. The results of the analysis are shown in Table 3. Furthermore, correlation analyses between each item of the Novaco Anger Scale, and three unsatisfied needs showed the trait anger was relevant to unsatisfied relatedness, autonomy, and competence (for details, see Supplementary Table 1). Therefore, it is unlikely that a unique relationship between trait anger and unsatisfied
Table 1 Descriptive statistics for psychological measures

\begin{tabular}{llllll}
\hline Scales & Mean & $S D$ & Range & Skewness & Kurtosis \\
\hline Relatedness dissatisfaction & 13.42 & 4.00 & $7-5$ & .75 & -.88 \\
Autonomy dissatisfaction & 16.72 & 4.36 & $8-26$ & .50 & .17 \\
Competence dissatisfaction & 15.65 & 4.46 & $9-29$ & .32 & .62 \\
Trait anger & 27.02 & 8.33 & $11-49$ & .26 & .31 \\
\hline
\end{tabular}

relatedness was only a byproduct of the scale being biased toward anger caused by deficits in social cognitive skills. Therefore, unsatisfied relatedness is likely the only factor that was uniquely associated with trait anger.

To further elucidate the neural basis of how unsatisfied relatedness leads to trait anger, we first replicated the previous finding regarding the association between trait anger and the amygdala. To do this, we conducted a partial correlation analysis on the GMV of the left and right amygdala. The results showed that trait anger was negatively correlated with the right amygdala GMV $(r=-.43, p=.001)$, but not with the left amygdala GMV ( $r=-.16, p=.25)$, after controlling for age, sex, and whole-brain GMV. However, the difference in correlation coefficients between left and right amygdala GMV was not significant (Steiger's $Z=-1.55, p=.12$, one-tailed). In addition, unsatisfied relatedness was negatively correlated with the right amygdala GMV $(r=-.42, p=.002)$, but not with the left amygdala GMV $(r=-.07, p=.63)$, after controlling for age, sex, whole-brain GMV, and unsatisfied autonomy and competence. Furthermore, the correlation coefficient between unsatisfied relatedness and right amygdala GMV was significantly larger than that of the left amygdala GMV (Steiger's $Z=-1.96, p<.05$, one-tailed). In short, the right amygdala was correlated with both trait anger and unsatisfied relatedness. Next, we examined whether the right amygdala mediated the relation between unsatisfied relatedness and trait anger.

We performed mediation analysis to examine whether unsatisfied relatedness exerted an influence on trait anger through the right amygdala GMV, with age, sex, wholebrain GMV, and unsatisfied autonomy and competence as irrelevant covariates. The results of the mediation analysis showed that the total effect of unsatisfied relatedness on trait anger was significant $(\beta=.40, p<.01)$. However, the effect of

Table 2 Correlations between needs dissatisfaction and trait anger

\begin{tabular}{llll}
\hline Scales & 1 & 2 & 3 \\
\hline 1 Relatedness dissatisfaction & 1 & & \\
2 Autonomy dissatisfaction & $.34^{* *}$ & 1 & \\
3 Competence dissatisfaction & $.43^{* *}$ & $.42^{* *}$ & 1 \\
4 Trait anger & $.53^{* * *}$ & $.42^{* *}$ & $.35^{* *}$ \\
\hline
\end{tabular}

$* * p<.01 . * * * p<.001$ 
Table 3 Results of multiple regressions predicting trait anger

\begin{tabular}{ll}
\hline Predictors & $\beta$ \\
\hline Sex & .02 \\
Age & -.02 \\
Relatedness dissatisfaction & $.42 * *$ \\
Autonomy dissatisfaction & .25 \\
Competence dissatisfaction & .06 \\
$R^{2}$ & .35 \\
\hline$* *<.01$ &
\end{tabular}

unsatisfied relatedness on trait anger became nonsignificant ( $\beta=.25, p=.07$ ) after the right amygdala was added as a mediator in the model. Bootstrap simulation $(n=5,000)$ further confirmed that the indirect effects on trait anger $(95 \% \mathrm{CI}$ $[.04, .85], p<.05)$ were significant, suggesting that the right amygdala fully mediated the effect of unsatisfied relatedness on trait anger (see Fig. 1). Notably, the mediation effect of the right amygdala maintained significance after controlling for participants' Big-Five personality traits and PANAS (95\% CI $[.04, .58], p<.05)$, suggesting the mediating effect of the right amygdala was not influenced by general personality dispositions.

Besides, we performed another mediation analysis to examine an alternative model whether unsatisfied relatedness mediated the relationship between amygdala volume and anger. The analysis showed that unsatisfied relatedness did not significantly mediate the relation between amygdala volume and anger (95\% CI $[-.43, .03], p=.11)$, after controlling for sex, age, whole-brain GMV, and unsatisfied autonomy and competence as irrelevant covariates. This result further confirmed the role of the amygdala in mediating the relation between anger and unsatisfied relatedness.

Finally, to explore regions correlated with trait anger other than the amygdala, we performed a whole-brain regression analysis. We found that besides the right amygdala, the lateral occipital cortex and middle frontal gyrus were also associated with trait anger $(p<.05, k>200$ voxels, uncorrected; see Table 4 and Fig. 2). However, further mediation analyses showed that neither the lateral occipital cortex $(95 \% \mathrm{CI}[-.14, .13], p=.94)$ nor the middle frontal gyrus $(95 \% \mathrm{CI}[-.26, .06], p=.26)$ mediated the relationship between unsatisfied relatedness and trait anger, after controlling for age, sex, whole-brain GMV, and unsatisfied autonomy and competence.

\section{Discussion}

The present study aimed to investigate how three basic psychological needs - relatedness, competence, and autonomywere related to trait anger. Although unsatisfied relatedness, autonomy, and competence were all correlated with

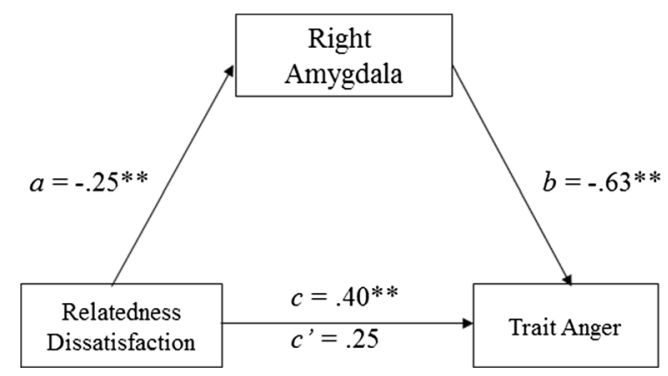

Fig. 1 Unsatisfied relatedness modulates trait anger through the right amygdala after controlling for sex, age, whole brain GMV, unsatisfied autonomy and competence. $* * p<.01$

participants' trait anger, a multiple-regression analysis indicated that unsatisfied relatedness accounted for the majority of the variance in trait anger, indicating that unsatisfied relatedness was the main source for trait anger among all the factors tested. In addition, we found that the right amygdala GMV was correlated with trait anger, consistent with previous neuroimaging studies that the amygdala serves as the core region for processing anger. Critically, the right amygdala totally mediated the association between unsatisfied relatedness and trait anger, even after controlling for general personality dispositions. Taken together, our study contributes to the theoretical conceptualization of anger by elucidating both behavioral and neural mechanisms underlying trait anger.

Behaviorally, we provided some of the first evidence that it was the unsatisfied relatedness, not autonomy or competence, that had the strongest correlation with trait anger. Although the items of Novaco Anger Scale were correlated with unsatisfied relatedness, competence, and autonomy, both unsatisfied autonomy and competence were not related to trait anger after removing the influence of unsatisfied relatedness, confirming the pivotal role of unsatisfied relatedness in predicting trait anger. This result supports the interpersonal hypothesis of anger that interpersonal rejection is the main determinant of anger (Leary et al., 2006). According to Baumeister and Leary's (1995) seminal work, the desire to feel belongingness is a fundamental human need that motivates people to ingratiate themselves with others. On the other hand, rejecting behavior from another individual may thwart a person's actions to meet the general need for acceptance or belongingness, reduce their

Table 4 Brain regions correlated with the trait anger by whole-brain analysis after controlling for age, sex, and whole brain GMV

\begin{tabular}{lllllll}
\hline \multirow{2}{*}{ Cluster } & Cluster size (voxels) & $t$ value & \multicolumn{2}{l}{ MNI coordinate } \\
\cline { 4 - 7 } & & & $x$ & $y$ & $z$ \\
\hline Right amygdala & 321 & 3.8 & 16 & -2 & -18 \\
Lateral occipital cortex & 303 & 3.39 & -36 & -84 & 22 \\
Middle frontal gyrus & 207 & 3.51 & -44 & 12 & 50 \\
\hline
\end{tabular}

Note: Only clusters surviving $(p<.05)$ for extent and greater than 200 voxels were reported 


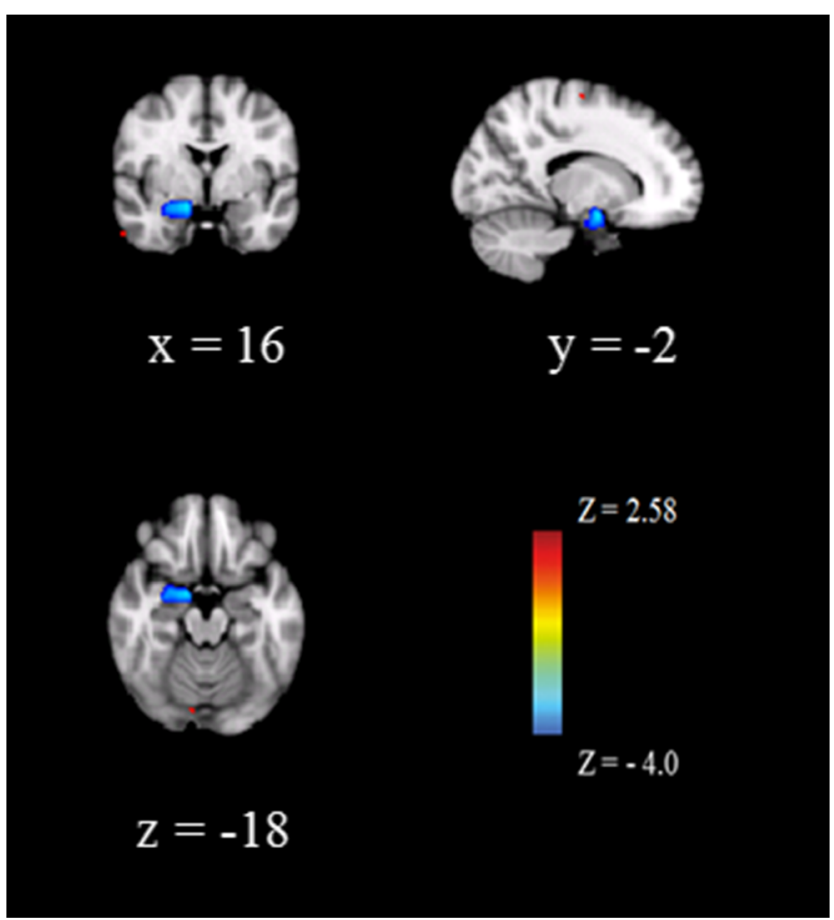

Fig. 2 Anger-related brain regions revealed by whole-brain analysis, after controlling for age, sex, and whole brain GMV $(p<.05$, uncorrected) (Color figure online)

relatedness satisfaction, and increase the likelihood of anger and aggression (Leary \& Baumeister, 2000; Leary, Tambor, Terdal, \& Downs, 1995) through threatened ego (Baumeister, Smart, \& Boden, 1996). Meanwhile, there is growing evidence showing that people often become angry when they feel devalued or outright rejected (Buckley, Winkel, \& Leary, 2004; Leary, Koch, \& Hechenbleikner, 2001; Williams, Cheung, \& Choi, 2000).

Further, for the first time our study revealed the right amygdala as the neural substrate linking unsatisfied relatedness with trait anger. We found that the right amygdala GMV was negatively correlated with trait anger. Because previous studies also show that the right amygdala is involved in processing perceived threats (Blair, 2012; Carlson et al., 2010), and low relatedness satisfaction is associated with threatened ego (Baumeister et al., 1996), it is not surprising that the right amygdala was also found to be related to unsatisfied relatedness, which further mediated its relation to anger.

Although anger has been theorized to be an approachrelated emotion and more associated with the left hemisphere (Harmon-Jones \& Allen, 1998), a study indicates that trait anger is also related to withdrawal behaviors through the right amygdala (Carlson et al., 2010). That is, the left amygdala is likely involved in approach-related behaviors (e.g., aggression; Bobes et al., 2013), whereas the right amygdala may be associated with withdrawal behaviors and negatively valenced perceptual processing (e.g., fear; Carlson et al., 2010). Consistent with this idea, our study demonstrated that unsatisfied relatedness leads to anger, possibly because of the fear of social rejection, not aggression to others.

In contrast, although the lateral occipital cortex and middle frontal gyrus were found to be related to trait anger, no studies have shown these regions having any role in perceiving threats. In line with this, these regions appeared to have no effects in mediating the relationship between unsatisfied relatedness and trait anger. In short, our study provides a framework for understanding the causes of anger by identifying the role of the right amygdala in mediating the association between unsatisfied relatedness and trait anger, inviting future studies to elucidate the mechanism of anger in the context of interpersonal interaction.

In sum, the current study has both important theoretical and practical implications. Theoretically, our findings contribute to the understanding of both behavioral and neural mechanisms of anger. Practically, because anger is an emotional concomitant to the propensity for aggression, the identification of the major factor leading to anger provides a means of appeasing anger and alleviating its negative effects. However, two limitations restrict our study in addressing the exact nature and the origin of the association we observed. First, we used a cross-sectional study approach to examine the relationship between unsatisfied relatedness and trait anger. Ideally, longitudinal studies are better to illustrate causality between the amygdala, unsatisfied relatedness, and anger. Another limitation is that our study was mainly dependent on self-reported measurements of unsatisfied needs and trait anger, which are susceptible to social desirability and self-preference. Future work may advance our findings with more objective measures.

Acknowledgements This study was funded by the National Natural Science Foundation of China (31230031), the National Basic Research Program of China (2014CB846101), the National Natural Science Foundation of China (31221003, 31471067, and 31470055), the National Social Science Foundation of China (13\&ZD073,14ZDB160), and Changjiang Scholars Programme of China.

\section{Compliance with ethical standards}

Conflict of interest The authors declared that they had no conflicts of interest with respect to their authorship or the publication of this article.

\section{References}

Ashburner, J. (2007). A fast diffeomorphic image registration algorithm. NeuroImage, 38(1), 95-113.

Ashburner, J., \& Friston, K. J. (2000). Voxel-based morphometry-The methods. NeuroImage, 11(6), 805-821.

Ashburner, J., \& Friston, K. J. (2005). Unified segmentation. NeuroImage, 26(3), 839-851.

Baumeister, R. F., \& Leary, M. R. (1995). The need to belong: desire for interpersonal attachments as a fundamental human motivation. Psychological Bulletin, 117(3), 497-529. 
Baumeister, R. F., Smart, L., \& Boden, J. (1996). Relation of threatened egotism to violence and aggression: The dark side of high self-esteem. Psychological Review, 103(1), 5-33.

Beaver, J. D., Lawrence, A. D., Passamonti, L., \& Calder, A. J. (2008). Appetitive motivation predicts the neural response to facial signals of aggression. The Journal of Neuroscience, 28(11), 2719-2725.

Berkowitz, L. (1989). Frustration-aggression hypothesis: Examination and reformulation. Psychological Bulletin, 106(1), 59.

Blair, R. (2003). Neurobiological basis of psychopathy. The British Journal of Psychiatry, 182(1), 5-7.

Blair, R. (2012). Considering anger from a cognitive neuroscience perspective. Wiley Interdisciplinary Reviews: Cognitive Science, 3(1), 65-74.

Bobes, M. A., Ostrosky, F., Diaz, K., Romero, C., Borja, K., Santos, Y., \& Valdés-Sosa, M. (2013). Linkage of functional and structural anomalies in the left amygdala of reactive-aggressive men. Social Cognitive and Affective Neuroscience, 8(8), 928-936.

Buckley, K., Winkel, R., \& Leary, M. (2004). Emotional and behavioral responses to interpersonal rejection: Anger, sadness, hurt, and aggression. Journal of Experimental Social Psychology, 40(1), 14-28.

Carlson, J. M., Greenberg, T., \& Mujica-Parodi, L. R. (2010). Blind rage? Heightened anger is associated with altered amygdala responses to masked and unmasked fearful faces. Psychiatry Research: Neuroimaging, 182(3), 281-283.

Carver, C. S., \& Harmon-Jones, E. (2009). Anger is an approach-related affect: Evidence and implications. Psychological Bulletin, 135(2), 183

Coccaro, E. F., McCloskey, M. S., Fitzgerald, D. A., \& Phan, K. L. (2007). Amygdala and orbitofrontal reactivity to social threat in individuals with impulsive aggression. Biological Psychiatry, 62(2), 168-178.

Deci, E. L., \& Ryan, R. M. (2000). The "what" and "why" of goal pursuits: Human needs and the self-determination of behavior. Psychological Inquiry, 11(4), 227-268.

Dollard, J., Miller, N. E., Doob, L. W., Mowrer, O. H., \& Sears, R. R. (1939). Frustration and aggression. New Haven: Yale University Press.

Fischer, A. H., \& Roseman, I. J. (2007). Beat them or ban them: The characteristics and social functions of anger and contempt. Journal of Personality and Social Psychology, 93(1), 103.

Frijda, N. H., Kuipers, P., \& Ter Schure, E. (1989). Relations among emotion, appraisal, and emotional action readiness. Journal of Personality and Social Psychology, 57(2), 212.

Ge, Y., Grossman, R. I., Babb, J. S., Rabin, M. L., Mannon, L. J., \& Kolson, D. L. (2002). Age-related total gray matter and white matter changes in normal adult brain. Part I: volumetric MR imaging analysis. American Journal of Neuroradiology, 23(8), 1327-1333.

Good, C. D., Johnsrude, I. S., Ashburner, J., Henson, R. N., Fristen, K., \& Frackowiak, R. S. (2002, June 15-23). A voxel-based morphometric study of ageing in 465 normal adult human brains. Paper presented at the 5th IEEE EMBS International Summer School on Biomedical Imaging, Berder Island, France.

Gosling, S. D., Rentfrow, P. J., \& Swann, W. B., Jr. (2003). A very brief measure of the Big-Five personality domains. Journal of Research in Personality, 37(6), 504-528.

Harmon-Jones, E. (2004). On the relationship of frontal brain activity and anger: Examining the role of attitude toward anger. Cognition and Emotion, 18(3), 337-361.

Harmon-Jones, E., \& Allen, J. J. (1998). Anger and frontal brain activity: EEG asymmetry consistent with approach motivation despite negative affective valence. Journal of Personality and Social Psychology, 74(5), 1310
Leary, M. R., \& Baumeister, R. F. (2000). The nature and function of selfesteem: Sociometer theory. Advances in Experimental Social Psychology, 32, 1-62. doi:10.1016/S0065-2601(00)80003-9

Leary, M. R., Koch, E. J., \& Hechenbleikner, N. R. (2001). Emotional responses to interpersonal rejection. In M. R. Leary (Ed.), Interpersonal rejection (pp. 145-166). Oxford: Oxford University Press.

Leary, M. R., Tambor, E. S., Terdal, S. K., \& Downs, D. L. (1995). Selfesteem as an interpersonal monitor: The sociometer hypothesis. Journal of Personality and Social Psychology, 68(3), 518.

Leary, M. R., Twenge, J. M., \& Quinlivan, E. (2006). Interpersonal rejection as a determinant of anger and aggression. Personality and Social Psychology Review, 10(2), 111-132.

Lemaitre, H., Crivello, F., Grassiot, B., Alpérovitch, A., Tzourio, C., \& Mazoyer, B. (2005). Age-and sex-related effects on the neuroanatomy of healthy elderly. NeuroImage, 26(3), 900-911.

Marcoulides, G. A., \& Hershberger, S. L. (1997). Multivariate statistical methods: A first course. New York: Psychology Press.

Mills, J. F., Kroner, D. G., \& Forth, A. E. (1998). Novaco Anger Scale: Reliability and validity within an adult criminal sample. Assessment, 5(3), 237-248.

Novaco, R. W. (1994). Anger as a risk factor for violence among the mentally disordered. In J. Monahan \& H. J. Steadman (Eds.), Violence and mental disorder: Developments in risk assessment (pp. 21-59). Chicago: University of Chicago Press.

Preacher, K. J., \& Hayes, A. (2008). Asymptotic and resampling strategies for assessingand comparing indirect effects in multiple mediator models. Behavior Research Methods, 40, 879-891.

Reuter, M., Weber, B., Fiebach, C. J., Elger, C., \& Montag, C. (2009). The biological basis of anger: Associations with the gene coding for DARPP-32 (PPP1R1B) and with amygdala volume. Behavioural Brain Research, 202(2), 179-183.

Roseman, I. J., \& Smith, C. A. (2001). Appraisal theory: Overview, assumptions, varieties, controversies. In R. Scherer, A. Schorr, \& T. Johnstone (Eds.), Appraisal processes in emotion: Theory, methods, and research (pp. 3-19). New York: Oxford University Press.

Rüsch, N., Van Elst, L. T., Ludaescher, P., Wilke, M., Huppertz, H.-J., Thiel, T., . . . Hesslinger, B. (2003). A voxel-based morphometric MRI study in female patients with borderline personality disorder. NeuroImage, 20(1), 385-392.

Sheldon, K. M., \& Gunz, A. (2009). Psychological needs as basic motives, not just experiential requirements. Journal of Personality, 77(5), 1467-1492.

Sheldon, K. M., \& Hilpert, J. C. (2012). The Balanced Measure of Psychological Needs (BMPN) scale: An alternative domain general measure of need satisfaction. Motivation and Emotion, 36(4), 439 451.

Smith, C. A., Haynes, K. N., Lazarus, R. S., \& Pope, L. K. (1993). In search of the "hot" cognitions: Attributions, appraisals, and their relation to emotion. Journal of Personality and Social Psychology, 65(5), 916

Tisserand, D. J., Van Boxtel, M. P., Pruessner, J. C., Hofman, P., Evans, A. C., \& Jolles, J. (2004). A voxel-based morphometric study to determine individual differences in gray matter density associated with age and cognitive change over time. Cerebral Cortex, 14(9), 966-973.

Watson, D., Clark, L. A., \& Tellegen, A. (1988). Development and validation of brief measures of positive and negative affect: The PANAS scales. Journal of Personality and Social Psychology, 54(6), 1063.

Williams, K. D., Cheung, C. K., \& Choi, W. (2000). Cyberostracism: Effects of being ignored over the Internet. Journal of Personality and Social Psychology, 79(5), 748. 